\title{
The chloroplasts genomic analyses of Rosa laevigata, R. rugosa and R. canina
}

\author{
Xianmei Yin ${ }^{1 \dagger}$, Baosheng Liao ${ }^{2 \dagger}$, Shuai Guo ${ }^{2}$, Conglian Liang ${ }^{3}$, Jin Pei ${ }^{1}$, Jiang $\mathrm{Xu}^{2^{*}}$ and Shilin Chen ${ }^{2}$
}

\begin{abstract}
Background: Many species of the genus Rosa have been used as ornamental plants and traditional medicines. However, industrial development of roses is hampered due to highly divergent characteristics.

Methods: We analyzed the chloroplast (cp) genomes of Rosa laevigata, $R$. rugosa and $R$. canina, including the repeat sequences, inverted-repeat (IR) contractions and expansions, and mutation sites.

Results: The size of the cp genome of R. laevigata, R. rugosa and R. canina was between 156333 bp and 156533 bp, and contained 113 genes (30 tRNA genes, 4 rRNA genes and 79 protein-coding genes). The regions with a higher degree of variation were screened out (trnH-GUU, trnS-GCU, trnG-GCC, psbA-trnH, trnC-GCA, petN, trnT-GGU, psbD, petA, psbJ, ndhF, rp/32,psaC and $n d h E)$. Such higher-resolution loci lay the foundation of barcode-based identification of $\mathrm{cp}$ genomes in Rosa genus. A phylogenetic tree of the genus Rosa was reconstructed using the full sequences of the $\mathrm{cp}$ genome. These results were largely in accordance with the current taxonomic status of Rosa.

Conclusions: Our data: (i) reveal that cp genomes can be used for the identification and classification of Rosa species; (ii) can aid studies on molecular identification, genetic transformation, expression of secondary metabolic pathways and resistant proteins; (iii) can lay a theoretical foundation for the discovery of disease-resistance genes and cultivation of Rosa species.
\end{abstract}

Keywords: Rosa species, cp genome, Phylogeny

\section{Background}

Rosaceae is a large and diverse family with 100 genera and 3000 species. Rosa is a typical genus of the Rosaceae family. Rosa chinensis Jacq., Rosa laevigata Michx. and Rosa rugosa Thunb are documented in the 2015 version of Chinese Pharmacopoeia [1].

Plants of the genus Rosa are distributed in the temperate and subtropical regions of the Northern hemisphere $[2,3]$. The genus Rosa has garnered increasing attention as a medicinal agent recently [4-6]. Due to the potential economic and medicinal value of peonies, it is important

\footnotetext{
*Correspondence: jxu@icmm.ac.cn

${ }^{+}$Xianmei Yin and Baosheng Liao contributed equally to this work ${ }^{2}$ Key Laboratory of Beijing for Identification and Safety Evaluation of Chinese Medicine, Institution of Chinese Materia Medica, China Academy of Chinese Medical Sciences, Beijing 100700, China

Full list of author information is available at the end of the article
}

to understand the genetic relationships within species for future application of germplasm resources.

In conventional taxonomy, the genus Rosa is divided into four subgenera (Hulthemia, Rosa, Platyrhodon, and Hesperhodos), and the subgenus of Rosa is divided further into 10 sections (Pimpinellifoliae, Gallicanae, Caninae, Carolinae, Rosa, Synstylae, Chinenses [syn. Indicae], Banksianae, Laevigatae, and Bracteatae [7, 8].

Despite numerous recent studies examining phylogenetic relationships in the genus Rosa, relationships remain obscure because of: (i) hybridization in nature and in the garden, and low levels of chloroplast and nuclear genome variation [9-11]; (ii) phylogenetic analyses only based on a small number of non-coding chloroplast sequences show low internal resolution $[12,13]$. Rosa laevigata, $R$. rugosa and $R$. canina have been employed in traditional Chinese medicine (TCM)

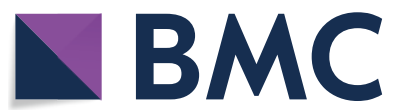

(c) The Author(s) 2020. This article is licensed under a Creative Commons Attribution 4.0 International License, which permits use, sharing, adaptation, distribution and reproduction in any medium or format, as long as you give appropriate credit to the original author(s) and the source, provide a link to the Creative Commons licence, and indicate if changes were made. The images or other third party material in this article are included in the article's Creative Commons licence, unless indicated otherwise in a credit line to the material. If material is not included in the article's Creative Commons licence and your intended use is not permitted by statutory regulation or exceeds the permitted use, you will need to obtain permission directly from the copyright holder. To view a copy of this licence, visit http://creativeco mmons.org/licenses/by/4.0/. The Creative Commons Public Domain Dedication waiver (http://creativecommons.org/publicdomain/ zero/1.0/) applies to the data made available in this article, unless otherwise stated in a credit line to the data. 
formulations. However, several sympatric species of Rosa have been used in TCM formulations, and the diversity of medicinal materials can affect the quality and safety of medicinal materials severely.

Chloroplasts are the descendants of ancient bacterial endosymbionts. They are the common organelles of green plants, and have an essential role in photosynthesis [14]. In general, inheritance of the cp genome is patrilineal in gymnosperms, but maternal in angiosperms [15]. The cp genome is conservative in structure, contains a large single-copy (LSC) region, small single-copy (SSC) region, and two inverse repeat (IR) regions. The cp genome is an ideal research model for the study of molecular identification, phylogeny, species conservation, and genome evolution [16, 17]. Over the last decade, researchers have gained more in-depth understanding of chloroplasts, including their origin, structure, evolution, genetic engineering, as well as forward and reverse genetics [18-20]. In addition, the development of sequencing technology has greatly promoted chloroplast study $[21,22]$, now generating massive chloroplast genome sequence data, helping to overcome the previously unresolved relationships. Moreover, it also provides genomic information such as structure, gene order, content, and mutations in which the critical information of species identification is provided [23-27].

In previous studies, chloroplast genomes provided the effective information for identifying Rosa species [28, 29]. The chloroplast genomes of two species from the genus Rosa, $R$. chinensis and $R$. rugosa, which have been collected in Chinese Pharmacopoeia 2015 were published. In the present study, the remainder of the recorded species of the genus Rosa in the Chinese Pharmacopeia, including two used in TCM ( $R$. laevigata, $R$. rugosa) and a traditional medicine used worldwide ( $R$. canina) were identified based on the chloroplast genome. The structural characteristics, phylogenetic relationships, interspecific divergence among $R$. laevigata, $R$. rugosa and $R$. canina were documented.

\section{Materials and methods \\ DNA sequencing, assembly and validation of the $\mathrm{cp}$ genome}

The fresh leaves of $R$. laevigata and $R$. rugosa plants were collected in Shennongjia (Hubei Province, China). The dried flowers of $R$ canina were purchased at a medicinal market in Beijing, China. The cetyltrimethylammonium-bromide method was used to extract the whole genomic DNA of tree peonies [30]. The DNA concentration was measured using a ND-2000 spectrometer (NanoDrop Technologies, Wilmington, DE, USA). A shotgun library (250 bp) was constructed according to manufacturer (Vazyme Biotech, Nanjing, China) instructions.
Sequencing was accomplished with the $\mathrm{X}^{\mathrm{TM}}$ Ten platform (Illumina, San Diego, CA, USA) using the double terminal sequencing method (pair-end 150). The amount of raw data from the sample was $5.0 \mathrm{G}$, and $>34$ million paired-end reads were attained.

Raw data were filtered by Skewer-0.2.2 [31]. Chloroplast-like reads were predicted from clean-reads by BLAST [32] searches using the sequences of the reference Rosa chinensis. Then, the cp reads was used to assemble sequences by SOAPdenovo-2.04 [33]. Finally, sequences were extended and gaps filled with SSPACE-3.0 and GapCloser-1.12 [34, 35]. To validate the accuracy of junction splicing, random primers were designed to test the four junctions of the sequence by polymerase chain reaction.

\section{Gene annotation and sequence analyses}

Sequence annotation was achieved by CpGAVAS [36]. DOGMA (http://dogma.ccbb.utexas.edu/) and BLAST were used to check the results of annotation [37]. All transfer tRNA genes with default settings were detected by tRNAscanSEv1. 21 [38]. The structural features of the cp genome were drawn by OGDRAWv1.2 [39]. MEGA5.2 was used to define relative use of synonymous codons [40].

\section{Comparison of cp genomes}

The cp genomes of Rosa species were completed by mVISTA [41] (Shuffle-LAGAN mode) using the genome of $R$. chinensis as the reference. Tandem Repeats Finder [42] was used to detect tandem repeats, forward repeats, and palindromic repeats as tested by REPuter [43]. Detection of simple sequence repeats (SSRs) was done by Misa.pl [44] using search parameters of mononucleotides set to $\geq 10$ repeat units, dinucleotides $\geq 8$ repeat units, trinucleotides and tetranucleotides $\geq 4$ repeat units, and pentanucleotides and hexanucleotides $\geq 3$ repeat units.

\section{Phylogenetic analyses}

Phylogenetic trees were constructed using the genomic sequences of 21 chloroplasts. The sequences were aligned using clustalw2. Construction of an unrooted phylogenetic tree was achieved using the neighbor-joining (NJ) approach with MEGA5.2 [40] with bootstrap replicates of 1000. Hibiscus rosa- sinensis was set as the outgroup.

\section{Results}

DNA features of the chloroplasts of R. laevigata, R. rugosa and $R$. canina

The size of the cp genomes ranged from $156333 \mathrm{bp}$ to $156533 \mathrm{bp}$. Among them, the largest cp genome was of $R$. rugosa (156 $533 \mathrm{bp}$ ) and the smallest cp genome was of $R$. laevigata (156 $333 \mathrm{bp}$ ). The total guanine + cytosine $(\mathrm{G}+\mathrm{C})$ content of the three genomes was $37.3 \%$. 
$R$. laevigata, $R$. rugosa and $R$. canina had a cp genome with a similar structure: LSC region, SSC region, and a pair of inverted repeats (IRA/IRB). For $R$. laevigata, $R$. rugosa and $R$. canina, the length of the LSC region of the cp genome varied from $85452 \mathrm{bp}$ to $85657 \mathrm{bp}$, and the $\mathrm{G}+\mathrm{C}$ content from $35.2 \%$ to $35.3 \%$; the length of SSCregion distribution was from $18742 \mathrm{bp}$ to $18785 \mathrm{bp}$, and the $\mathrm{G}+\mathrm{C}$ content was from 31.3 to $31.4 \%$. The IR region had a length distribution from $26048 \mathrm{bp}$ to $26053 \mathrm{bp}$, and the $\mathrm{G}+\mathrm{C}$ content was $42.7 \%$ (Table 1). The DNA $\mathrm{G}+\mathrm{C}$ content is an important indicator of species affinity [45], and $R$. laevigata, $R$. rugosa and $R$. canina have highly similar cpDNA G $+C$ content. The DNA G $+C$ content of the IR regions was higher than that of LSC and SSC regions, which is similar to that seen with other angiosperms [46]. In general, the relatively high DNA G+C content of the IR regions is attributable to rRNA genes and tRNA genes $[47,48]$. After annotation, the sequences of the whole cp genome of $R$. laevigata, $R$. rugosa and $R$. canina was submitted to the National Center for Biotechnology Information database (NCBI), the GenBank accession number in Table 1.

A physical map of the cp genomes of $R$. laevigata, $R$. rugosa and $R$. canina was drawn according to annotation results using OGDraw [39] (Fig. 1). A total of 113 genes were contained in the cp genome of $R$. laevigata, $R$. rugosa and $R$. canina: four rRNA genes, 30 tRNA genes, and 79 protein-coding genes (Table 2). Most genes could be divided crudely into three groups: "self-replicationrelated", "photosynthesis-related", and "other" (Table 2) [49].

Table 1 Summary of complete chloroplast chloroplast genomes for $R$. laevigata, $R$. rugosa and $R$. canina

\begin{tabular}{|c|c|c|c|}
\hline Species & R. rugosa & R. laevigata & R. cania \\
\hline \multicolumn{4}{|l|}{ Large single copy } \\
\hline Length (bp) & 85,657 & 85,452 & 85,653 \\
\hline$G+C(\%)$ & 35.2 & 35.3 & 35.2 \\
\hline Length (\%) & 54.7 & 54.7 & 54.7 \\
\hline \multicolumn{4}{|l|}{ Small single copy } \\
\hline Length (bp) & 18,780 & 18,785 & 18,742 \\
\hline$G+C(\%)$ & 31.4 & 31.3 & 31.4 \\
\hline Length (\%) & 12.0 & 12.0 & 12.0 \\
\hline \multicolumn{4}{|l|}{ Inverse repeat } \\
\hline Length (bp) & 26,048 & 26,048 & 26,053 \\
\hline$G+C(\%)$ & 42.7 & 42.7 & 42.7 \\
\hline Length (\%) & 16.7 & 16.7 & 16.7 \\
\hline \multicolumn{4}{|l|}{ Total } \\
\hline Length (bp) & 156,533 & 156,333 & 156,501 \\
\hline$G+C(\%)$ & 37.3 & 37.3 & 37.3 \\
\hline Accession number & MN661138 & MN661139 & MN661140 \\
\hline
\end{tabular}

In all anticipated genes of the cp genomes of $R$. laevigata, $R$. rugosa and $R$. canina, introns were discovered in 17 genes: six tRNA genes and 11 protein-encoding genes (Table 3). The tRNA genes with introns were trnK$U U U$, trnL-UAA, trnV-UAC, trnI-GAU, trnG-UCC and trnA-UGC. The 11 coding genes with introns were rps12, rps16, rpl16, rpl2, rpoC1, ndhA, ndhB, ycf3, petB, clpP and petD. Three of the 17 intron-containing genes were inserted by three introns (rps12, ycf3, $\operatorname{clpP}$ ). The remainder of the genes were inserted by only one intron. Of these, trnH-UUU contained the largest intron (2500 bp), which contained the whole matK. Similar to other angiosperms, rps12 of chloroplasts in $R$. laevigata, $R$. rugosa and $R$. canina resulted from trans-splicing activity. The $5^{\prime}$ end of rps 12 was in the LSC region, and the $3^{\prime}$ end was in the IR region.

\section{Analyses of long repetitive sequences and SSRs}

For $R$. laevigata, $R$. rugosa and $R$. canina, interspersed repeated sequences (IRSs) were evaluated in the $\mathrm{cp}$ genomes with a repeat-unit length of $\geq 30 \mathrm{bp}$. These comprised forward repeats, reverse repeats, complementary repeats, and palindromic repeats. Fifty 50 IRSs were found in R. rugosa; 60 IRS in R. laevigata and 50 IRS in $R$. canina. Among all types of IRS, the sequence lengths of 20-29 bp occurred most frequently. IRS analyses of the cp genomes of $R$. laevigata, $R$. rugosa and $R$. canina are shown as Fig. 2.

SSRs are disposed to slipped-strand mispairing, which is a key mutational mechanism for generating SSR polymorphisms [50]. SSRs at the intra-specific level in the cp genome are variable, so they are used regularly as genetic markers in studies of evolution and population genetics [51-53]. We found 63 SSRs in $R$. rugosa, 62 SSRs in $R$ canina, and 65 SSRs in R. laevigata (Fig. 3).

\section{Genomic sequences}

To ascertain differences in the genomic sequences of chloroplasts of $R$. laevigata, $R$. rugosa and $R$. canina, we used the sequence in $R$. chinensis as a reference (Fig. 4). Variability in the IR region of the cp genomes was considerably lower than that of LSC and SSC regions. In addition, most of the protein-coding genes of chloroplasts were highly conserved, except for the large variation in protein-coding genes of some genes (e.g., rps19, $p e t B$, and $y c f 2$ ). Regions with a higher degree of variation among chloroplast genomic sequences were usually located in intergenic regions, such as the spacers for: trnH-GUU; trnS-GCU and trnG-GCC; $p s b A-\operatorname{trnH}$, trnC$G C A$ and petN; trnT-GGU and $p s b D$; petA and $p s b J ; n d h F$ and rpl32; $p s a C$ and $n d h E$. Identification of such higherresolution loci was necessary for use as barcodes for species identification. 


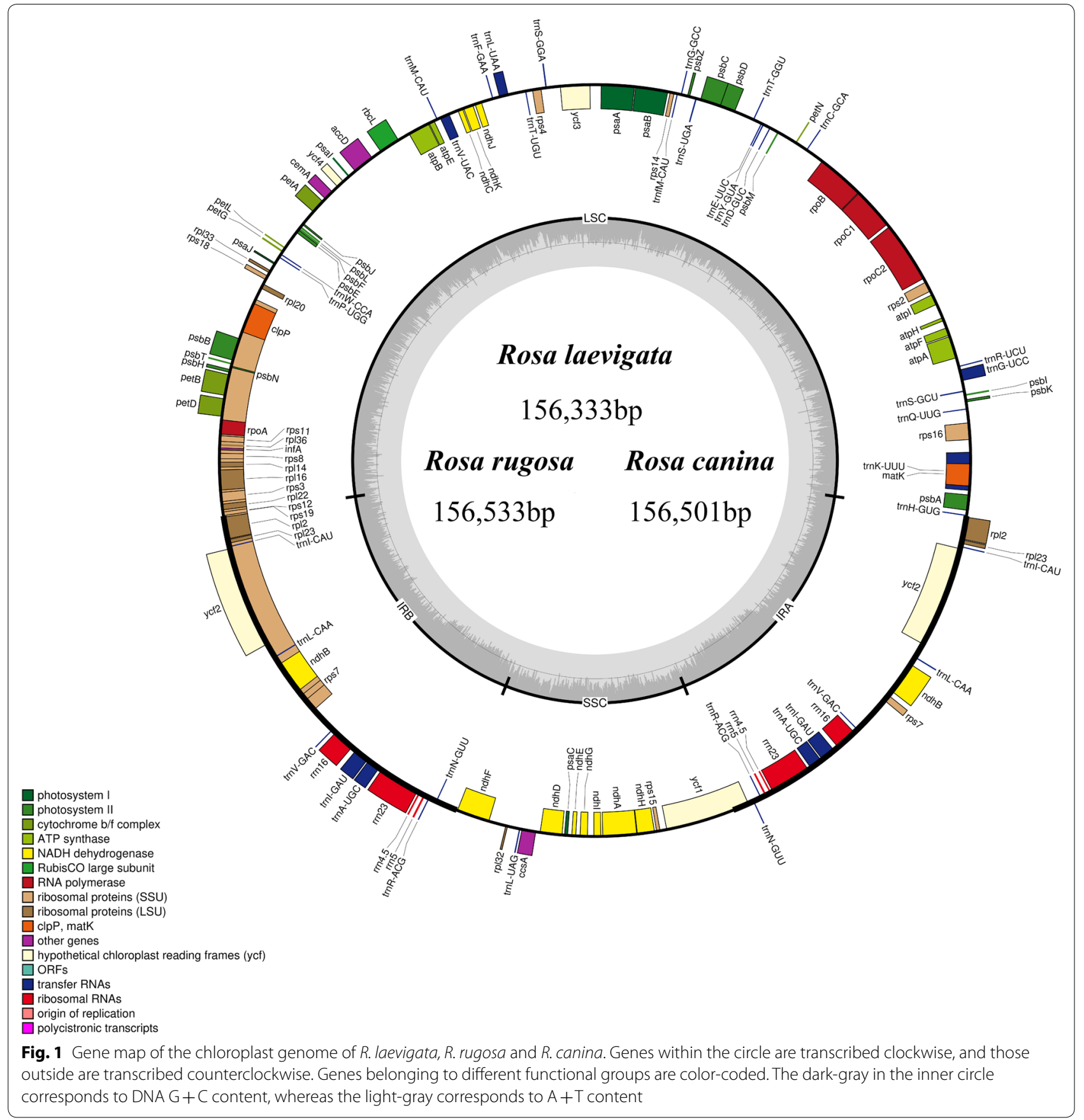

Comparison of IR regions in the cp genomes of $R$. laevigata, $R$. rugosa, $R$. canina and $R$. chinensis

Gene location was relatively conservative in $R$. laevigata, $R$. rugosa, $R$. canina and $R$. chinensis. In these four species, rps19 was located in the LSC region, rpl2 in the IRa region, and $n d h F$ in the SSC region. However, the coding region of $y c f 1$ was at the border of SSC/IRb, and spanned the LSC region and IRb region, so the IRa/
SSC boundary ( $5^{\prime}$ end was lost) region created a pseudogene. The region of mutations in the $y c f 1$ pseudogene in the IRa/SSC region was $1106-1118$ bp (Fig. 5). The double-strand break repair theory is considered to be the main mechanism for expansion and contraction of the IR region. Large shrinkages of the IR region are relatively rare. 
Table 2 Genes in the chloroplast genome of R. laevigata, R. rugosa and R. canina

\begin{tabular}{|c|c|c|}
\hline Category & Group of genes & Name of genes \\
\hline \multirow[t]{5}{*}{ Self-replication } & Large subunit of ribosomal proteins & $r p / 2 *, a, 14,16^{*}, 20,22,23 a, 33,36$ \\
\hline & Small subunit of ribosomal proteins & $\operatorname{rps} 2,3,4,7 a, 8,11,12^{*}, a, 14,16^{*}, 18,19$ \\
\hline & DNA-dependent RNA polymerase & $\operatorname{rpoA}, B, C 1^{*}, C 2$ \\
\hline & rRNA genes & rrn16Sa, rrn23Sa, rrn4.5Sa, rrn5Sa \\
\hline & tRNA genes & 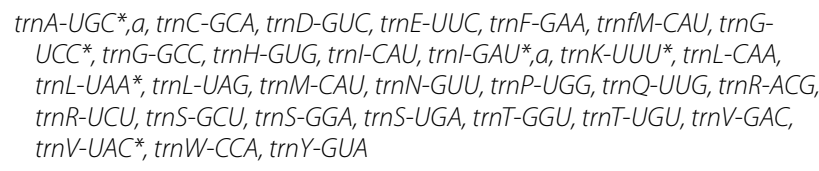 \\
\hline \multirow[t]{6}{*}{ Photosynthesis } & Photosystem I & psaA, $B, C, I, J$ \\
\hline & Photosystem II & $p s b A, B, C, D, E, F, H, I, J, K, L, M, N, T, Z$, \\
\hline & NADH oxidoreductase & $n d h A^{*}, B^{*}, a, C, D, E, F, G, H, I, J, K$ \\
\hline & Cytochrome b6/f complex & $\operatorname{pet} A, B^{*}, D^{*}, G, L, N$ \\
\hline & ATP synthase & $\operatorname{atp} A, B, E, F^{*}, H, l$ \\
\hline & Rubisco & $r b c L$ \\
\hline \multirow[t]{6}{*}{ Other genes } & Maturase & matk \\
\hline & Protease & $c l p P^{*}$ \\
\hline & Envelope membrane protein & $\operatorname{cem} A$ \\
\hline & Subunit acetyl-CoA-carboxylase & $a c c D$ \\
\hline & c-Type cytochrome synthesis gene & $\operatorname{cCs} A$ \\
\hline & Conserved open-reading frames & $y c f 1,2 a, 3^{*}, 4,15$ \\
\hline
\end{tabular}

\section{Phylogenetic analyses}

There have been many efforts to reconstruct the phylogenetic trees of plants of the genus Rosa. Several scholars have proposed that the extant classification system was artificial [12, 13], and that the interspecies relationships of Rosa are still ambiguous. The availability of the complete genomes of chloroplasts can provide further information for reconstruction of robust phylogeny for Rosa. A NJ tree was constructed for the cp genomes of 18 species of the Rosaceae family (Fig. 6). Species from the Rosa genus were monophyletic clade. Furthermore, $R$. laevigata, $R$. rugosa, $R$. canina and $R$. chinensis could be effectively divided into different sub-clades, and differentiated from each other efficiently. In which, $R$. Chinensis have a closer relationship with R. rugosa.

\section{Discussion}

We identified the cp genomes of $R$. laevigata, $R$. rugosa and $R$. canina in this study, which are used in TCM formulations. The cp genomes of $R$. laevigata, $R$. rugosa and $R$. canina showed high similarities in terms of genome size, gene classes, gene sequences, codon usage, and distribution of repeat sequences. This is partly because of the extremely low levels of sequence divergence observed across the Rosa genus [54, 55]. Some intergenic regions were identified with high degree of variation, which will be used as barcodes for species identification. We also investigated introns in all anticipated genes of three Rosa species. Intron and/or gene losses have been reported for cp genomes [56-58]. Introns have important roles in regulation of gene expression [59], and they can control gene expression temporally and in a tissue-specific manner $[60,61]$. Scholars have reported on the regulation mechanisms of introns for gene expression in plants and animals [62-64]. However, the connotations between intron loss and gene expression using the transcriptome for genus Rosa have not been published. More experimental work on the roles of introns shall be needed for future work. Comparative analysis of gene location in $R$. laevigata, $R$. rugosa, $R$. canina and $R$. chinensis revealed a pseudogene of $y c f 1$, which may provide a basis for studying variations in the cp genomes of higher plants or algae.

Phylogenetic analyses revealed that Rosa genus belonged to monophyletic clade (Fig. 6), while their intrafamily relationships were almost in agreement with those from a study by Zhang and Marie et al. [13, 65]. However, the exact phylogenetic location of some base taxons needs further verification, such as that the phylogenetic relationship of $R$. rugosa and $R$. chinensis in here contradicts what was previously reported, two $R$. rugosa species were clustered into two different clades. The possible reason is: complicates phylogeny reconstruction in roses was complicated by interspecific hybridization, some studies have suggested that there were frequent interspecific hybridization in the Rosa genus [11, 66-69]. Indeed, several contradictions between plastid and nuclear gene 
Table 3 Length of exons and introns in genes with introns in the chloroplast genome of three medicinal roses

\begin{tabular}{|c|c|c|c|c|c|c|c|}
\hline Species & Gene & Location & Exon I (bp) & Intron I (bp) & Exon II (bp) & Intron II (bp) & Exon III (bp) \\
\hline \multirow[t]{17}{*}{ R. rugosa } & $\operatorname{trnK}$-UUU & LSC & 35 & 350 & 37 & & \\
\hline & $\operatorname{trnG}-U C C$ & LSC & 23 & 698 & 48 & & \\
\hline & $\operatorname{trn} L-U A A$ & LSC & 35 & 549 & 50 & & \\
\hline & $\operatorname{trnV} V-U A C$ & LSC & 35 & 600 & 39 & & \\
\hline & $\operatorname{trn} l-G A U$ & $\mathbb{R}$ & 37 & 956 & 35 & & \\
\hline & $\operatorname{trn} A-U G C$ & $\mathbb{R}$ & 38 & 814 & 35 & & \\
\hline & $r p s 12^{*}$ & LSC & 26 & 538 & 232 & & 114 \\
\hline & rps16 & LSC & 230 & 881 & 40 & & \\
\hline & $r p / 16$ & LSC & 399 & 980 & 9 & & \\
\hline & $r p / 2$ & $\mathbb{R}$ & 434 & 683 & 391 & & \\
\hline & rpocl & LSC & 1611 & 769 & 453 & & \\
\hline & $n d h A$ & SSC & 540 & 1216 & 552 & & \\
\hline & $n d h B$ & $\mathbb{R}$ & 756 & 678 & 777 & & \\
\hline & $y c f 3$ & SSC & 153 & 768 & 228 & 742 & 126 \\
\hline & petB & LSC & 6 & 791 & 642 & & \\
\hline & $c l p P$ & LSC & 228 & 647 & 292 & 844 & 71 \\
\hline & petD & LSC & 8 & 722 & 475 & & \\
\hline \multirow[t]{17}{*}{ R. laevigata } & $\operatorname{trnK-UUU}$ & LSC & 35 & 2500 & 37 & & \\
\hline & $\operatorname{trnG}-U C C$ & LSC & 23 & 697 & 48 & & \\
\hline & $\operatorname{trn} L-U A A$ & LSC & 35 & 549 & 50 & & \\
\hline & $\operatorname{trnV} V-U A C$ & LSC & 35 & 600 & 39 & & \\
\hline & trnl-GAU & $\mathbb{I R}$ & 37 & 956 & 35 & & \\
\hline & $\operatorname{trn} A-U G C$ & $\mathbb{R}$ & 38 & 816 & 35 & & \\
\hline & $\operatorname{rps} 12^{*}$ & LSC & 26 & 538 & 232 & & 114 \\
\hline & rps16 & LSC & 230 & 872 & 40 & & \\
\hline & $r p / 16$ & LSC & 399 & 964 & 9 & & \\
\hline & $r p / 2$ & $\mathbb{R}$ & 434 & 683 & 391 & & \\
\hline & rpoCl & LSC & 1611 & 769 & 453 & & \\
\hline & $n d h A$ & SSC & 540 & 1245 & 522 & & \\
\hline & $n d h B$ & $\mathbb{R}$ & 756 & 678 & 777 & & \\
\hline & ycf3 & SSC & 153 & 782 & 228 & 742 & 126 \\
\hline & petB & LSC & 6 & 793 & 642 & & \\
\hline & $c l p P$ & LSC & 228 & 648 & 292 & 831 & 71 \\
\hline & petD & LSC & 8 & 722 & 475 & & \\
\hline \multirow[t]{17}{*}{ R. cania } & $\operatorname{trnK-UUU}$ & LSC & 35 & 2500 & 37 & & \\
\hline & $\operatorname{trnG}-U C C$ & LSC & 23 & 697 & 48 & & \\
\hline & $\operatorname{trn} L-U A A$ & LSC & 35 & 548 & 50 & & \\
\hline & $\operatorname{trn} V-U A C$ & LSC & 35 & 600 & 39 & & \\
\hline & trnl-GAU & $\mathbb{R}$ & 37 & 956 & 35 & & \\
\hline & $\operatorname{trnA-UGC}$ & $\mathbb{I R}$ & 38 & 814 & 35 & & \\
\hline & $\operatorname{rps} 12^{*}$ & LSC & 26 & 538 & 232 & & 114 \\
\hline & $r p s 16$ & LSC & 230 & 883 & 40 & & \\
\hline & $r p / 16$ & LSC & 399 & 964 & 9 & & \\
\hline & $r p / 2$ & $\mathbb{R}$ & 434 & 683 & 391 & & \\
\hline & rpoCl & LSC & 1611 & 700 & 453 & & \\
\hline & $n d h A$ & SSC & 540 & 1205 & 552 & & \\
\hline & $n d h B$ & $\mathbb{R}$ & 756 & 678 & 777 & & \\
\hline & $y c f 3$ & SSC & 153 & 785 & 228 & 740 & 126 \\
\hline & petB & LSC & 6 & 792 & 642 & & \\
\hline & $c \mid p P$ & LSC & 228 & 650 & 292 & 839 & 71 \\
\hline & petD & LSC & 8 & 728 & 475 & & \\
\hline
\end{tabular}




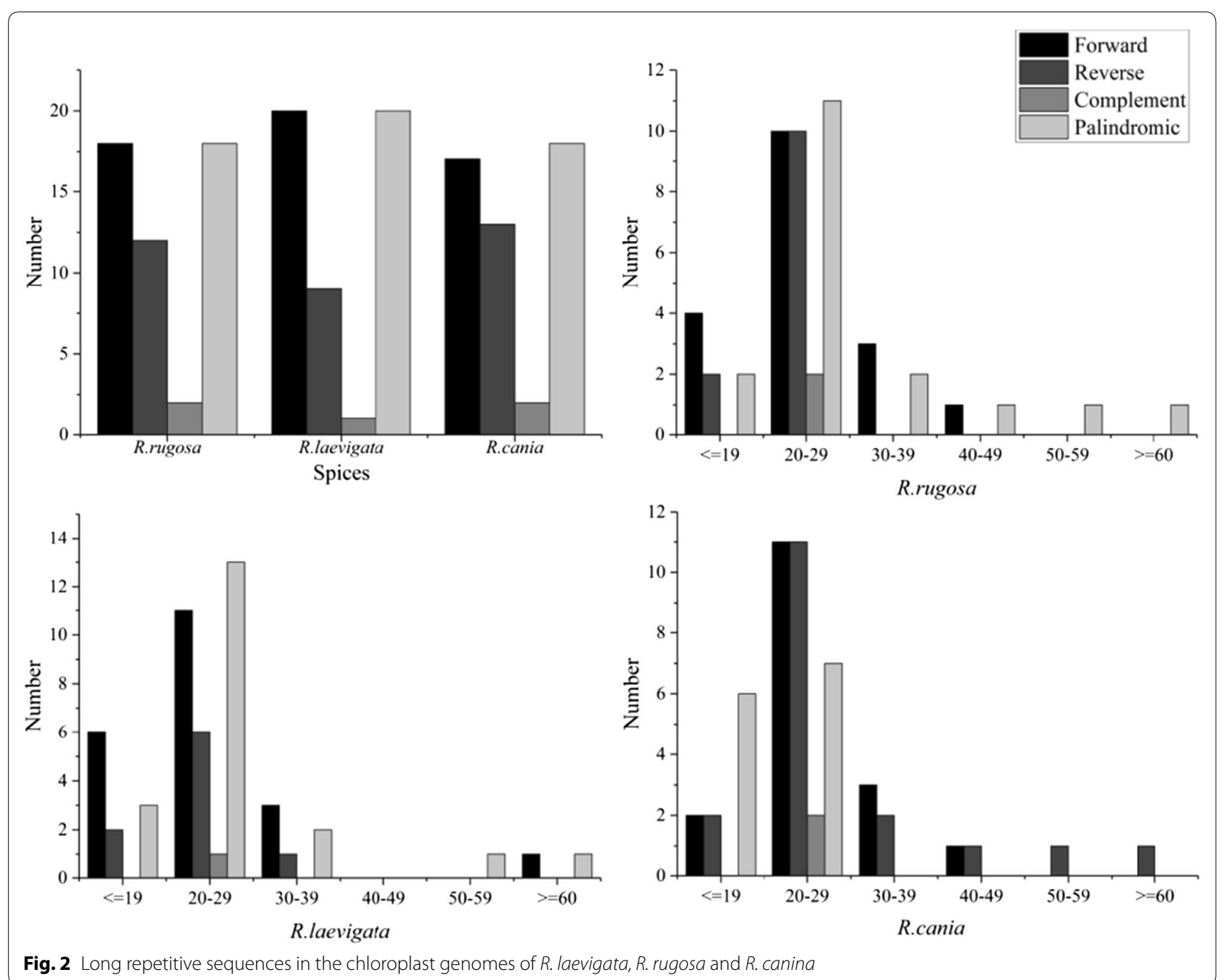

Fig. 2 Long repetitive sequences in the chloroplast genomes of R. laevigata, R. rugosa and R. canina

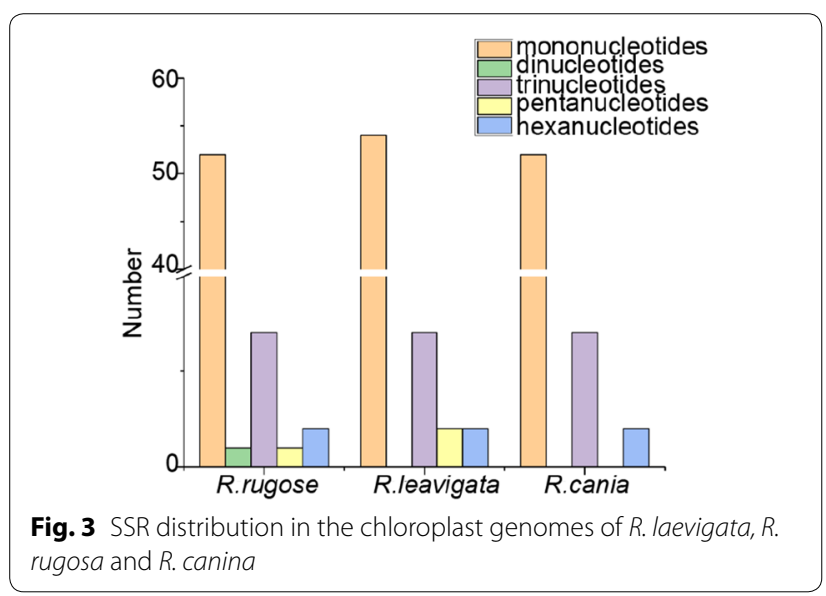

phylogenies of Rosa genus were discovered in previous study [55]. In addition, publications of numerous names given to morphological variants and hybrids, result in
Rosa taxonomy further complication [70]. Further identification of plant material or sequencing of those hybrids could explain why conspecific samples sometimes fall into distinct clades [12].

\section{Conclusions}

The whole cp genomes of $R$. laevigata, $R$. rugosa and $R$. canina was sequencing and analysis in this study. The status of the major taxa within the genus Rosa was consistent with our results for sequencing of cp genomes. $R$. laevigata, $R$. rugosa, $R$. canina and $R$. chinensis could be differentiated from other Rosa species efficiently. Our data reveal that cp genomes can be used for the identification and classification of Rosa species. Our results can aid studies on molecular identification, genetic transformation, and lay a theoretical foundation for the discovery of disease-resistance genes and cultivation of Rosa 

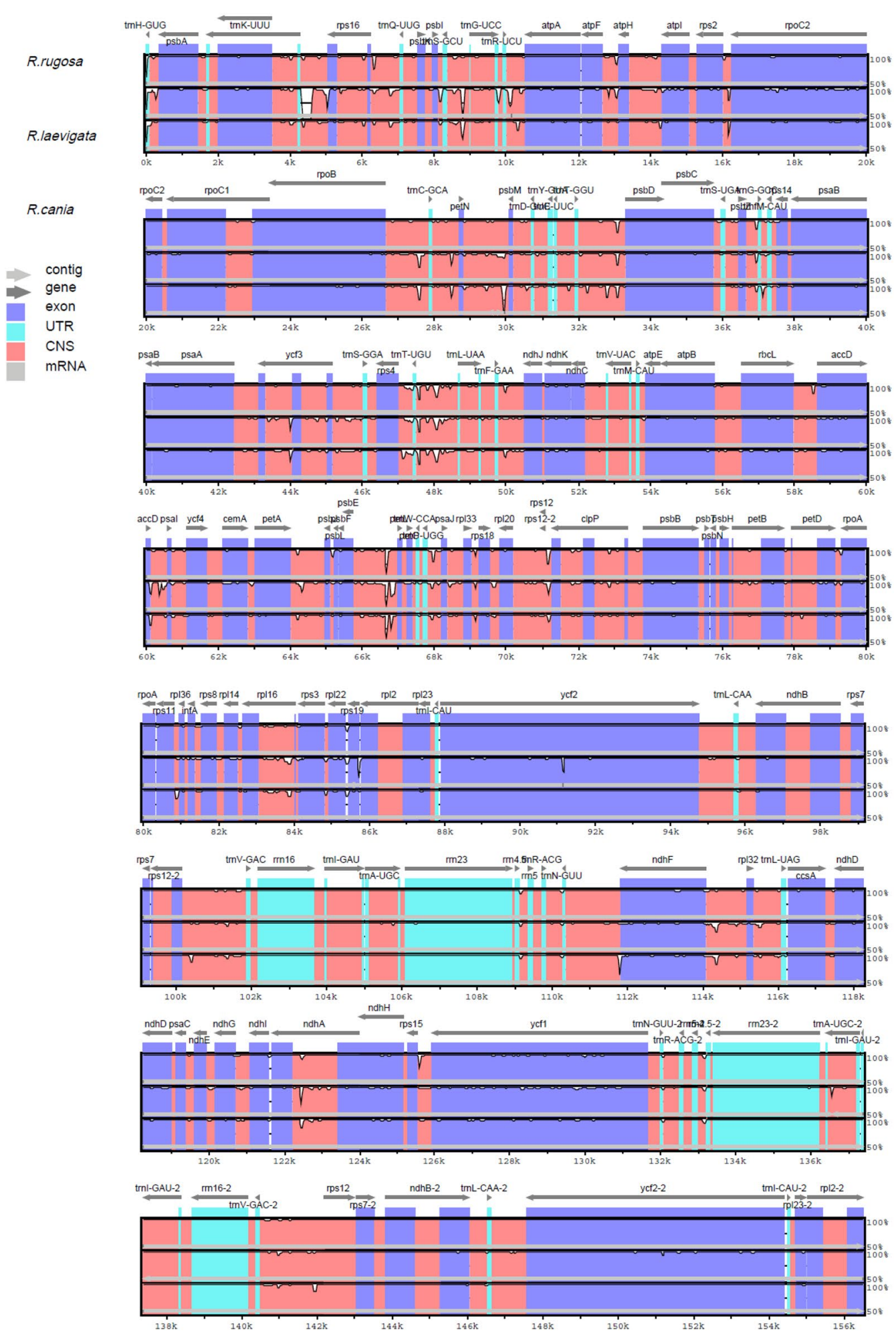

Fig. 4 Comparative analyses of genomic differences in chloroplasts of R. laevigata, R. rugosa and R. canina. Gray arrows and thick black lines above the alignment indicate gene orientation. Purple bars represent exons, blue bars denote untranslated regions (UTRs), pink bars represent non-coding sequences (CNS) and gray bars denote mRNA. The $y$-axis represents the percentage identity 


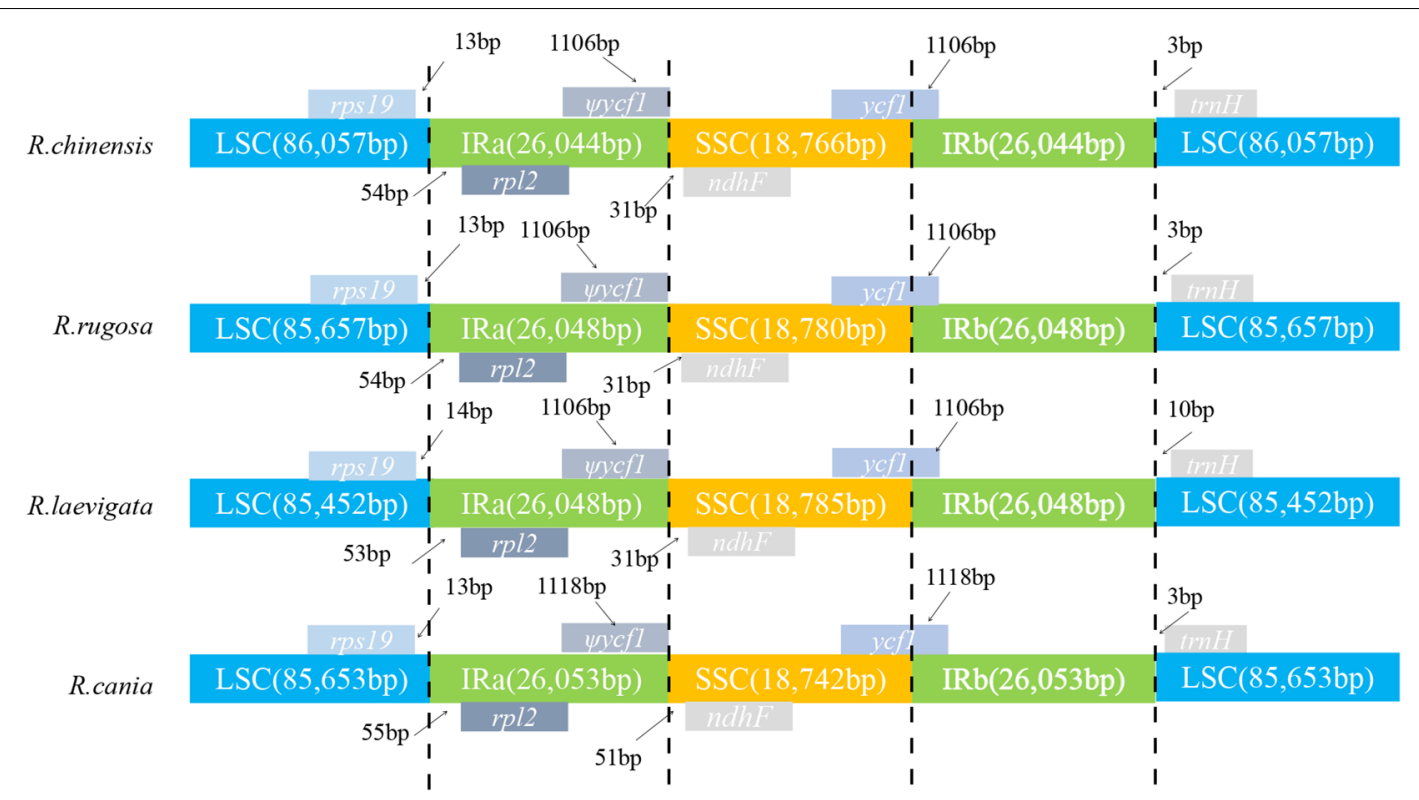

Fig. 5 Comparison of genome boundaries in chloroplasts from R. laevigata, R. rugosa, R. canina and R. chinensis

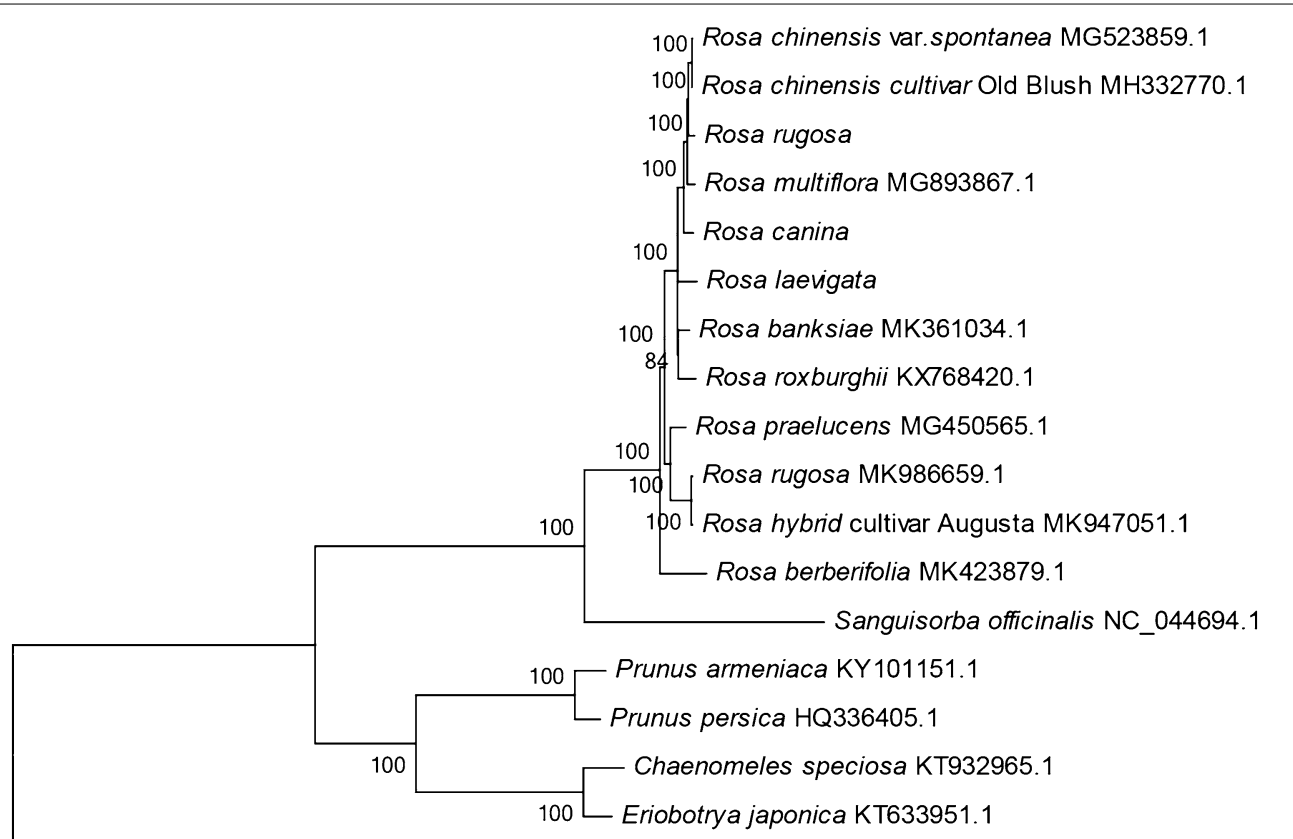

Hibiscus rosa-sinensis MK382984.1

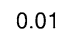

Fig. $6 \mathrm{NJ}$ tree based on the cp genomes of the Rosaceae family. Hibiscus rosa-sinensis was set as the outgroup

species. Our observations complement the database of herbgenomics [71].
Abbreviations

LSC: Large single-copy; SSC: Small single-copy region; IR: Inverse repeat; cD: Chloroplast; NCBI: National Center for Biotechnology Information database; TCM: Traditional Chinese medicine. 


\section{Acknowledgements}

I sincerely thank all those who provided an abundance of helpful on my data analysis and tried their best to improve my paper.

\section{Authors' contributions}

JX conceived and designed the research framework; JP collected and identified the sample; $X Y, S G$ and $B L$ performed the experiments; $X Y, B L$ and $C L$ analyzed the data; and $X Y$ and $B L$ wrote the paper. $S C$ made revisions to the final manuscript. All the authors read and approved the final manuscript.

\section{Funding}

The Major Project of "Research on modernization of traditional Chinese medicine", under Grant \#2017YFC1702100.

\section{Availability of data and materials}

The datasets generated during the current study are available in the National Center for Biotechnology Information database (NCBI). https://www.ncbi.nlm. nih.gov/[MN661138, MN661139, MN661140].

\section{Ethics approval and consent to participate}

Not applicable.

\section{Consent for publication}

Not applicable.

\section{Competing interests}

The authors declare that they have no competing interests.

\section{Author details}

${ }^{1}$ College of Pharmacy, Chengdu University of Traditional Chinese Medicine, Chengdu 611130, China. ${ }^{2}$ Key Laboratory of Beijing for Identification and Safety Evaluation of Chinese Medicine, Institution of Chinese Materia Medica, China Academy of Chinese Medical Sciences, Beijing 100700, China. ${ }^{3}$ College of Pharmacy, Shandong University of Traditional Chinese Medicine, Jinan 250355, China.

Received: 10 December 2019 Accepted: 5 February 2020

Published online: 13 February 2020

\section{References}

1. Anonymous. Pharmacopoeia of People's Republic of China. Part 2. Chinese Medicine. Edited by Committee NP. Beijing: Science Press., 2015;75:200, 221.

2. Wissemann V, Ritz CM. The genus Rosa (Rosoideae, Rosaceae) revisited: molecular analysis of nrlTS- 1 and atpB-rbcL intergenic spacer (IGS) versus conventional taxonomy. Bot J Linn Soc. 2005;147(3):275-90.

3. Christenhusz MJM, Fay MF, Chase MW. Plants of the world: an illustrated encyclopedia of vascular plants. Madroño. 2018;65(2):101-2.

4. Uzunçakmak T, Ayaz Alkaya S. Effect of aromatherapy on coping with premenstrual syndrome: a randomized controlled trial. Complement Ther Med. 2017:36:63-7.

5. Baiyisaiti A, Wang Y, Zhang $X$, et al. Rosa rugosa flavonoids exhibited PPARa agonist-like effects on genetic severe hypertriglyceridemia of mice. J Ethnopharmacol. 2019;240:111952.

6. Liu Y, Zhi D, Wang X, Fei D, et al. Rose (R Setate $\times$ R. Rugosa) decoction exerts antitumor effects in C. elegans by down regulating Ras/MAPK pathway and resisting oxidative stress. Int J Mol Med. 2018;42(3):1-8.

7. Rehder A. Bibliography of cultivated trees and shrubs hardy in the cooler temperate regions of the Northern Hemisphere, vol. 1. Jamaica Plain: Arnold Arboretum of Harvard University; 1949. p. 296-317.

8. Zhu ZM, Gao XF, Fougère Danezan M. Phylogeny of Rosa sections Chinenses and Synstylae (Rosaceae) based on chloroplast and nuclear markers. Mol Phylogenet Evol. 2015;87:50-64.

9. Matthews JR. Hybridism and classification in the genus rosa. New Phytol. 1920;19(7-8):153-71.

10. Rowley G. Some naming problems in rosa. Bulletin du Jardin botanique de l'Etat Bruxelles. 1959;29(3):205-11.
11. Ritz CM, Schmuths H, Wissemann V. Evolution by reticulation: European Dogroses originated by multiple hybridization across the genus Rosa. J Hered. 2005;96(1):4-14.

12. Joly S, Starr JR, Bruneau A. Phylogenetic relationships in the genus Rosa: new evidence from chloroplast DNA sequences and an appraisal of current knowledge. Syst Bot. 2007;32(2):366-78.

13. Fougère-Danezan $M$, Simon J, Anne $B$, et al. Phylogeny and biogeography of wild roses with specific attention to polyploids. Ann Bot. 2015;115(2):275-91.

14. Brunkard JO, Runkel AM, Zambryski PC. Chloroplasts extend stromules independently and in response to internal redox signals. Proc Natl Acad Sci. 2015;112(32):10044-9.

15. Hu Y, Zhang Q, Rao G. Occurrence of plastids in the sperm cells of Caprifoliaceae: biparental plastid inheritance in angiosperms is unilaterally derived from maternal inheritance. Plant Cell Physio. 2008;49(6):958-68.

16. Daniell H, Kumar S, Dufourmantel N. Breakthrough in chloroplast genetic engineering of agronomically important crops. Trends Biotechnol. 2005:23(5):238-45.

17. Sigmon BA, Adams RP, Mower JP. Complete chloroplast genome sequencing of vetiver grass (Chrysopogon zizanioides) identifies markers that distinguish the non-fertile 'Sunshine' cultivar from other accessions. Ind Crops Prod. 2017;108:629-35.

18. Kim KJ, Lee HL. Complete chloroplast genome sequence from Korean Ginseng (Panax schiseng Nees) and comparative analysis of sequence evolution among 17 vascular plants. DNA Res. 2004;11(4):247-61.

19. Li P, Zhang S, Li F, et al. A phylogenetic analysis of chloroplast genomes elucidates the relationships of the six economically important brassica species comprising the triangle of U. Front Plant Sci. 2017;8:111.

20. Shen X, Wu M, Liao B, et al. Complete chloroplast genome sequence and phylogenetic analysis of the medicinal plant Artemisia annua. Molecules. 2017:22(8):1330.

21. Li R, Ma PF, Wen J, Yi TS. Complete sequencing of five Araliaceae chloroplast genomes and the phylogenetic implications. PLOS ONE. 2013;8(10):e78568.

22. Yang JB, Li DZ, Li HT, et al. Highly effective sequencing whole chloroplast genomes of angiosperms by nine novel universal primer pairs. Mol Ecol Resour. 2014;14(5):1024-31.

23. Kane N, Sveinsson S, Dempewolf H, et al. Ultra-barcoding in cacao (Theobroma spp; Malvaceae) using whole chloroplast genomes and nuclear ribosomal DNA. Am J Bot. 2012;99(2):320-9.

24. Dodsworth Steven. Genome skimming for next-generation biodiversity analysis. Trends Plant Sci. 2015;20(9):525-7.

25. Wang A, Wu H, Zhu X, et al. Species identification of Conyza bonariensis assisted by chloroplast genome sequencing. Front Genet. 2018;9:374.

26. Luo H, Shi J, Arndt W, et al. Gene Order Phylogeny of the genus Prochlorococcus. PLOS ONE. 2008:3(12):e3837.

27. Luo H, Sun Z, Arndt W, et al. Gene order phylogeny and the evolution of methanogens. PLoS ONE. 2009;4(6):e6069.

28. Jeon JH, Kim SC. Comparative analysis of the complete chloroplast genome sequences of three closely related east-asian wild roses (rosa sect. synstylae; rosaceae). Genes. 2019;10(1):23.

29. Jiang HY, Zhang YH, Yan HJ, et al. The complete chloroplast genome of a key ancestor of modern roses, rosa chinensis var. spontanea, and a comparison with congeneric species. Molecules. 2018;23(2):389.

30. Guo Q, Guo LL, Zhang L, et al. Construction of a genetic linkage map in tree peony (Paeonia Sect. Moutan) using simple sequence repeat (SSR) markers. Sci Hortic. 2017;219:294-301.

31. Jiang $H$, Lei $R$, Ding SW, et al. Skewer: a fast and accurate adapter trimmer for next-generation sequencing paired-end reads. BMC Bioinform. 2014;15:182.

32. Deng P, Wang L, Cui L, et al. Global identification of microRNAs and their targets in Barley under salinity stress. PLOS ONE. 2015;10(9):e0137990

33. Gogniashvili M, Naskidashvili P, Bedoshvili D, et al. Complete chloroplast DNA sequences of Zanduri wheat (Triticum s). Genet Resour Crop Evol. 2015;62:1269-77.

34. Acemel RD, Tena JJ, Irastorza-Azcarate I, et al. A single threedimensional chromatin compartment in amphioxus indicates a stepwise evolution of vertebrate hox bimodal regulation. Nat Genet. 2016:48(3):336-41. 
35. Boetzer M, Henkel CV, Jansen $\mathrm{HJ}$, et al. Scaffolding pre-assembled contigs using SSPACE. Bioinformatics. 2010;27(4):578-9.

36. Liu C, Shi L, Zhu Y, et al. CpGAVAS, an integrated web server for the annotation, visualization, analysis, and GenBank submission of completely sequenced chloroplast genome sequences. BMC Genomics. 2012;13(1):715-22.

37. Wyman SK, Jansen RK, Boore JL. Automatic annotation of organellar genomes with DOGMA. Bioinformatics. 2004;20(17):3252-5.

38. Lowe TM, Eddy SR. tRNAscan-SE: a program for improved detection of transfer RNA genes in genomic sequence. Nucleic Acids Res. 1997;25(5):955-64.

39. Lohse M, Drechsel O, Bock R. OrganellarGenomeDRAW (OGDRAW): a tool for the easy generation of high-quality custom graphical maps of plastid and mitochondrial genomes. Curr Genet. 2007;52(5-6):267-74.

40. Tamura K, Peterson D, Peterson N, et al. MEGA5: molecular evolutionary genetics analysis using maximum likelihood, evolutionary distance, and maximum parsimony methods. Mol Biol Evol. 2011;28(10):2731-9.

41. Frazer KA, Pachter L, Poliakov A, et al. VISTA: computational tools for comparative genomics. Nucleic Acids Res. 2004;32(Web Server issue):W273-9.

42. Benson G. Tandem repeats finder: a program to analyze DNA sequences. Nucleic Acids Res. 1999;27(2):573-80.

43. Kurtz S, Choudhuri JV, Ohlebusch E, et al. REPuter: the manifold applications of repeat analysis on a genomic scale. Nucleic Acids Res. 2001:29(22):4633-42.

44. Beier S, Thiel T, Münch T, et al. MISA-web: a web server for microsatellite prediction. Bioinformatics. 2017;33(16):2583-5

45. Choi KS, Park S. The complete chloroplast genome sequence of Aster spathulifolius (Asteraceae); genomic features and relationship with Asteraceae. Gene. 2015;572(2):214-21.

46. Guo S, Guo L, Zhao W, et al. Complete chloroplast genome sequence and phylogenetic analysis of Aster tataricus. Molecules. 2018:23(10):246.

47. Doorduin L, Gravendeel B, Lammers Y, et al. The complete chloroplast genome of 17 individuals of pest species Jacobaea vulgaris: SNPs, microsatellites and barcoding markers for population and phylogenetic studies. DNA Res. 2011;18(2):93-105

48. Lee SB, Kaittanis C, Jansen RK, et al. The complete chloroplast genome sequence of Gossypium hirsutum: organization and phylogenetic relationships to other angiosperms. BMC Genomics. 2006;7:61.

49. Saski C, Lee S, Daniell H, et al. Complete chloroplast genome sequence of Glycine max and comparative analyses with other legume genomes. Plt Mol Biol. 2005;59(2):309-22.

50. Asaf S, Khan AL, Khan MA, et al. chloroplast genomes of Arabidopsis halleri ssp. gemmifera and Arabidopsis lyrata ssp. petraea: structures and comparative analysis. Sci Rep. 2017;7(1):7556.

51. Dong W, Xu C, Cheng T, et al. Sequencing angiosperm plastid genomes made easy: a complete set of universal primers and a case study on the phylogeny of Saxifragales. Genome Biol Evol. 2013;5(5):989-97.

52. Yang Y, Zhou T, Duan D, et al. Comparative analysis of the complete chloroplast genomes of five Quercus species. Front Plant Sci. 2016;7:959.

53. Suo Z, Li W, Jin X, Zhang H. A new nuclear DNA marker revealing both microsatellite variations and single nucleotide polymorphic loci: a case study on classification of cultivars in Lagerstroemia indica L. J Microb Biochem Technol. 2016:8:266-71.
54. Matsumoto S, Kouchi M, Yabuki J, et al. Phylogenetic analyses of the genus Rosa using the matK sequence: molecu-lar evidence for the narrow genetic background of modern roses. Scientia Hortic. 1998; $77(1-2): 73-82$.

55. Wissemann V, Ritz CM. The genus Rosa (Rosoideae, Rosaceae) revisited: molecular analysis of $\mathrm{nr}$ ITS-1 and atpB-rbcL intergenic spacer(IGS) versus conventional taxonomy. Botanical J Linn Soc. 2005;147(3):275-90.

56. Downie SR, Llanas E, Katz-Downie DS. Multiple independent losses of the rpoC1 intron in angiosperm chloroplast DNAs. Syst Bot. 1996;21(2):135-51.

57. Graveley BR. Alternative splicing: increasing diversity in the proteomic world. Trends Genet. 2001;17(2):100-7.

58. Ueda M, Fujimoto M, Arimura SI, et al. Loss of the rpl32 gene from the chloroplast genome and subsequent acquisition of a preexisting transit peptide within the nuclear gene in Populus. Gene. 2007:402(1-2):51-6.

59. Xu J, Chu Y, Liao B, et al. Panax ginseng genome examination for ginsenoside biosynthesis. Gigascience. 2017;6(11):1-15.

60. Le Hir H, Nott A, Moore MJ. How introns influence and enhance eukaryotic gene expression. Trends Biochem Sci. 2003;28(4):215-20.

61. Niu DK, Yang YF. Why eukaryotic cells use introns to enhance gene expression: splicing reduces transcription-associated mutagenesis by inhibiting topoisomerase I cutting activity. Biol Direct. 2011;6:24.

62. Callis J, Fromm M, Walbot V. Introns increase gene expression in cultured maize cells. Genes Dev. 1987;1(10):1183-200.

63. Emami S, Arumainayagam D, Korf I, et al. The effects of a stimulating intron on the expression of heterologous genes in Arabidopsis thaliana. Plant Biotechnol J. 2013;11(5):555-63.

64. Ted C, Manley H, Cornelia G, et al. A generic intron increases gene expression in transgenic mice. Mol Cell Biol. 1991;11(6):3070-4.

65. Zhang SD, Jin JJ, Chen SY, et al. Diversification of Rosaceae since the Late Cretaceous based on plastid phylogenomics. New Phytol. 2017;214(3):1355-67.

66. Joly S, Starr JR, Lewis WH, et al. Polyploid and hybrid evolutionin roses east of the Rocky Mountains. Am J Bot. 2006;93(3):412-25.

67. Mercure M, Bruneau A. Hybridization between the escaped Rosa rugosa(Rosaceae) and native R. blanda in Eastern North America. Am J Bot. 2008;95(5):597-607.

68. Ritz CM, Koehnen I, Groth M, et al. To be or not to be the odd one outallele-specific transcription in pentaploid dogroses (Rosa L. sect. Caninae (DC) Ser). BMC Plant Biol. 2011;11(1):37.

69. Qiu XQ, Zhang H, Wang QG, et al. Phylogenetic relationships of wild roses in China based on nrDNA and matK data. Sci Hortic. 2012:140:45-51.

70. Wissemann V. Conventional taxonomy (wild roses). In: Roberts AV, Debener T, Gudin S, editors. Encyclopedia of rose science. Amsterdam: Elsevier; 2003. p. 111-7.

71. Hu H, Shen X, Liao B, et al. Herbgenomics: a stepping stone for research into herbal medicine. Sci China Life Sci. 2019:62(7):913-20.

\section{Publisher's Note}

Springer Nature remains neutral with regard to jurisdictional claims in published maps and institutional affiliations.

Ready to submit your research? Choose BMC and benefit from

- fast, convenient online submission

- thorough peer review by experienced researchers in your field

- rapid publication on acceptance

- support for research data, including large and complex data types

- gold Open Access which fosters wider collaboration and increased citations

- maximum visibility for your research: over $100 \mathrm{M}$ website views per year

At BMC, research is always in progress.

Learn more biomedcentral.com/submissions 